\title{
Filocrono, área foliar e produtividade de frutos de berinjela conduzidas com uma e duas hastes por planta em estufa plástica
}

\author{
Phyllochron, leaf area, and fruit yield in eggplants grown with one and two stems per plant \\ in plastic greenhouse
}

\author{
Ivan Carlos Maldaner ${ }^{\mathrm{I}}$ Fabrício Ivan Guse ${ }^{\mathrm{I}}$ Nereu Augusto Streck ${ }^{\mathrm{I} *}$ Arno Bernardo Heldwein ${ }^{\mathrm{II}}$ \\ Dionéia Daiane Pitol Lucas ${ }^{\text {III }}$ Luis Henrique Loose ${ }^{\text {III }}$
}

\section{RESUMO}

Os objetivos deste trabalho foram estimar o filocrono, relacionar a área foliar com o número de folhas e determinar a produtividade de frutos em plantas de três genótipos de berinjela conduzidas com uma e duas hastes dentro de estufa plástica. $O$ experimento foi conduzido na área experimental do Departamento de Fitotecnia da Universidade Federal de Santa Maria, Santa Maria, Rio Grande do Sul (RS). Foram utilizados três genótipos de berinjela: Nápoli, Comprida Roxa e Ciça. O transplante das mudas foi realizado com cinco folhas definitivas no dia 29 de outubro de 2007. O delineamento experimental foi o de blocos ao acaso com quatro repetições, compostas de duas fileiras de oito plantas. Em quatro plantas de cada repetição, foram realizadas observações do número de folhas na haste principal e na secundária. Em duas plantas de cada repetição, foi medida a largura das folhas na haste principal e na secundária. O filocrono foi estimado pelo inverso do coeficiente angular da regressão linear entre o número de folhas acumuladas na haste e a soma térmica acumulada após o transplante. A emissão de folhas na berinjela foi mais rápida nas plantas conduzidas com duas hastes. A área foliar da berinjela pode ser estimada por meio do número de folhas acumuladas. A produtividade de frutos de berinjela foi maior nas plantas conduzidas com duas hastes, sendo o genótipo Ciça aquele que apresentou maior produtividade de frutos.

Palavras-chave: aparecimento de folhas, soma térmica, área foliar, Solanum melongena $L$.

\section{ABSTRACT}

The objective of this study was to estimate the phyllochron, to associate the leaf area to the leaf number and to determine fruit yield of eggplants grown with one and two stems inside a plastic greenhouse. An experiment was carried at the experimental area of the Plant Science Department of the Federal University of Santa Maria, Santa Maria, RS, Brazil. Three eggplant genotypes were used: Nápoli, Comprida roxa, and Ciça. Five-leaf seedlings were transplanted on 10/29/2007. The experimental design was a complete randomized blocks with four replications composed by two rows with eight plants. The number of leaves on the main stem and secondary branches was counted in four plants of each replication. Leaf width was measured on two plants per replication. The phyllochron was estimated as the inverse of the slope of the linear regression between the leaf number and the accumulated thermal time after transplanting. Eggplant fruit yield was greater in plants grown with two stems than and Ciça had the higher fruit yield among genotypes. The time for leaves emergence in eggplant was reduced in plants with two stems than in plants with only the main stem. Leaf area of eggplant can be estimated as function of the accumulated number of leaves. Eggplant fruit yield was greater in plants conducted with two stems than with only the main stem, and Ciça presented the greatest yield among genotypes.

Key words: leaf appearance, thermal time, plant development, leaf area, Solanum melongena $L$..

\section{INTRODUÇÃO}

A berinjela (Solanum melongena L.) é uma solanácea originária de regiões tropicais do Oriente, sendo cultivada há muitos séculos por chineses e árabes. $\mathrm{O}$ aumento do consumo de berinjela tem sido

'Programa de Pós-graduação em Engenharia Agrícola, Universidade Federal de Santa Maria (UFSM), Santa Maria, RS, Brasil.

"Departamento de Fitotecnia, Centro de Ciências Rurais (CCR), UFSM, 97105-900, Santa Maria, RS, Brasil E-mail: nstreck2@yahoo.com.br.*Autor para correspondência.

II'Curso de Agronomia, CCR, UFSM, Santa Maria, RS, Brasil. 
motivado por uma procura maior por parte dos consumidores de produtos mais saudáveis e com propriedades medicinais. Nesse aspecto, a berinjela se destaca pela sua propriedade redutora do nível de colesterol (FILGUEIRA, 2000). Com o aumento da demanda, o cultivo da berinjela em ambientes protegidos vem se tornando uma alternativa viável, sendo uma fonte de renda para as pequenas propriedades rurais. Nesse sentido, essa cultura necessita ser mais estudada em ambiente protegido.

Parâmetros de desenvolvimento e crescimento vegetal estão relacionados com a produtividade da cultura, sendo processos independentes e sincronizados que podem ocorrer simultaneamente ou não (WILHELM \& McMASTER, 1995). A diferenciação celular, a iniciação e o aparecimento de órgãos até a senescência da cultura estão relacionados com o desenvolvimento vegetal, enquanto o aumento irreversível de uma grandeza física como massa, área, altura, diâmetro e volume compõem o crescimento vegetal (HODGES, 1991).

O desenvolvimento de plantas depende principalmente da temperatura do ar. A soma térmica acima de uma temperatura mínima ou base, com unidade ${ }^{\circ} \mathrm{C}$ dia, é a melhor medida de tempo (biológico realístico) para quantificar o efeito da temperatura sobre o desenvolvimento das plantas, em comparação com os dias do calendário civil (GILMORE \& ROGERS, 1958; RUSSELE et al., 1984).

Um parâmetro do desenvolvimento vegetal de particular interesse é o número de folhas acumuladas na haste principal (NF), que é o resultado da integração da taxa de aparecimento de folhas no tempo (STRECK et al., 2003). O NF está relacionado com o surgimento de alguns órgãos na planta e com a expansão da área foliar, a qual intercepta a radiação solar usada na fotossíntese para acúmulo de fitomassa (SINCLAIR et al., 2004; STRECK et al., 2005a, b). Para estimar o NF, pode-se utilizar o conceito do filocrono, que é o intervalo, em graus-dia, entre a emissão de folhas sucessivas, com unidade ${ }^{\circ} \mathrm{C}$ dia folha ${ }^{-1}$ (HERMES et al., 2001; XUE et al., 2004).

No cultivo de tomate em estufa plástica, a condução em uma haste apenas (haste principal) é o método mais utilizado no país (OLIVEIRA et al., 1995; SILVA et al., 1997), porém vários autores têm sugerido a condução das plantas com duas hastes (haste principal e uma haste secundária) que proporciona aumento na produtividade (FONTES et al., 1987; OLIVEIRA et al., 1995; POERSCHKE et al., 1995). O número de hastes por planta determina a área foliar para interceptação da radiação solar e realização da fotossíntese pelo dossel (CAMARGOS, 1998), mas também afeta a competição intra-específica por água, luz e nutrientes, o que pode afetar a produtividade de frutos. No entanto, não foram encontrados estudos relacionados à caracterização de parâmetros de crescimento e desenvolvimento quando as plantas de berinjela são conduzidas com uma e duas hastes. Também não foram encontrados na literatura estudos que quantificassem o filocrono em berinjela.

Os objetivos deste trabalho foram estimar o filocrono, relacionar a área foliar com o número de folhas e determinar a produtividade de frutos em plantas de três genótipos de berinjela conduzidas com uma e duas hastes em estufa plástica.

\section{MATERIAL E MÉTODOS}

O experimento foi realizado na área experimental do Departamento de Fitotecnia da Universidade Federal de Santa Maria, em Santa Maria, Rio Grande do Sul (RS). A cultura da berinjela foi cultivada em uma estufa tipo arco-pampeana com dimensões de 24 x 10m, coberta com filme de polietileno de baixa densidade (PEBD) de 0,15mm de espessura.

Foram utilizados três genótipos de berinjela, sendo dois híbridos (Napoli e Ciça) e um uma variedade (Comprida Roxa). A semeadura foi realizada em bandejas de isopor com 128 alvéolos no dia 05 de setembro de 2007, as quais permaneceram em uma casa de vegetação até a data do transplante. O transplante para o solo da estufa foi realizado no dia 29 de outubro de 2007, quando as mudas tinham cinco folhas verdadeiras, sobre camalhões de $20 \mathrm{~cm}$ de altura coberto com filme preto de PEBD, no espaçamento de 0,50 x $1,00 \mathrm{~m}$. O experimento terminou no dia 13 de fevereiro de 2008. A condução das plantas foi feita verticalmente com fio de ráfia. Nas plantas que foram conduzidas com uma haste, foi deixada a haste principal. Nas plantas conduzidas com duas hastes, além da haste principal, foi deixada uma haste secundária resultante do crescimento do broto lateral abaixo da primeira flor. Os demais brotos laterais foram retirados assim que apareciam. A irrigação foi realizada por gotejamento, e a adubação foi realizada com base nos resultados da análise do solo, sendo aplicados $28 \mathrm{~kg}$ de $\mathrm{N} \mathrm{ha}^{-1}$, 83,3kg de $\mathrm{P}_{2} \mathrm{O}_{5}$ ha $^{-1}$ e $125 \mathrm{~kg}$ de $\mathrm{K}_{2} \mathrm{O}$ ha $^{-1}, 10$ dias antes do transplante.

O delineamento experimental foi em blocos ao acaso, com quatro repetições. Cada parcela foi constituída de oito plantas em duas linhas de plantio. Em cada parcela foram marcadas quatro plantas, nas quais foi contado o número de folhas visíveis (NF), considerando-se visível as folhas com mais de $1 \mathrm{~cm}$ de comprimento, a data do início da floração (50\% das 
plantas com a primeira flor visível), a data do início da frutificação ( $50 \%$ das plantas com pelo menos um fruto com mais de $1 \mathrm{~cm}$ de diâmetro) e a data da primeira colheita (50\% das plantas com um fruto de coloração alterado do roxo para o lilás). Também foi contado o número final de folhas (NFF) em cada haste das plantas marcadas. O diâmetro de frutos foi medido com um paquímetro, e o comprimento de frutos foi medido com uma régua. A produtividade e o peso médio de frutos foram obtidos pela pesagem dos frutos de cada planta, separadamente. Em duas das plantas marcadas por parcela, foram realizadas medições semanais da largura de todas as folhas da planta para a determinação da área foliar pela equação (COSTA et al., 2007):

$\mathrm{AF}=0,8841 \times \mathrm{L}^{1,9862}$

em que: $\mathrm{AF}=$ área foliar em $\mathrm{cm}^{2} ; \mathrm{L}=$ largura da folha em cm.

As temperaturas mínima $\left(\mathrm{T}_{\min }\right)$ e máxima $\left(\mathrm{T}_{\text {máx }}\right)$ diárias do ar durante o período experimental foram medidas com um termômetro de mínima de álcool e um termômetro de máxima de mercúrio, respectivamente, instalados no interior de um abrigo meteorológico a $1,5 \mathrm{~m}$ de altura do solo, no centro da estufa. A temperatura média $\left(\mathrm{T}_{\text {média }}\right)$ diária foi calculada pela média aritmética entre a mínima e a máxima diária. Os graus-dia diários (GDD, ${ }^{\circ} \mathrm{C}$ dia) foram calculados por (GILMORE \& ROGERS, 1958, STRECK et al., 2005a):

GDD $=\left(\mathrm{T}_{\text {média }}-\mathrm{Tb}\right) * 1$ dia, quando $\mathrm{T}_{\mathrm{b}} \leq \mathrm{T}_{\text {média }} \leq \mathrm{T}_{\mathrm{ot}}(2)$ GDD $=\left(\left(\mathrm{T}_{\mathrm{ot}}-\mathrm{T}_{\mathrm{b}}\right) *\left(\mathrm{~T}_{\mathrm{B}}-\mathrm{T}_{\text {média }}\right)\right) /\left(\mathrm{T}_{\mathrm{B}}-\mathrm{T}_{\mathrm{ot}}\right) * 1$ dia, quando Tot $<$ Tmed $=$ TB

se $\mathrm{T}_{\text {media }}<\mathrm{T}_{\mathrm{b}}$ então $\mathrm{T}_{\text {media }}=\mathrm{T}_{\mathrm{b}}$ se $\mathrm{T}_{\text {med }}>\mathrm{T}_{\mathrm{B}}$ então $\mathrm{T}_{\text {media }}=\mathrm{T}_{\mathrm{B}}$ em que $\mathrm{T}_{\mathrm{b}}, \mathrm{T}_{\text {ot }}$ e $\mathrm{T}_{\mathrm{B}} \stackrel{\text { são as temperaturas }}{\text { en }}$ cardeais' (basal inferior, ótima e basal superior, respectivamente) para a berinjela. Foram usadas as temperaturas cardeais de $\mathrm{Tb}=10^{\circ} \mathrm{C}(\mathrm{UZUN}, 2006)$, $\mathrm{Tot}=$ $22^{\circ} \mathrm{C}$ (KÜRKLÜ et al., 1998; UZUN, 2006) e TB $=35^{\circ} \mathrm{C}$ (YI, 1999). A soma térmica acumulada (STa, ${ }^{\circ} \mathrm{C}$ dia) a partir do terceiro dia após o transplante foi calculada acumulando-se GDD, ou seja, STa $=\Sigma($ GDD). Optouse em começar a acumular GDD no terceiro dia após o transplante para não considerar o acúmulo térmico durante o possível estresse causado as plantas.

Foi realizada uma análise de regressão linear simples entre NF na haste principal e na haste secundária e STa em cada repetição (média de quatro plantas). O filocrono na haste principal e na secundária para cada genótipo foi estimado pelo inverso do coeficiente angular da regressão linear entre o NF e a STa (STRECK et al., 2005b). A análise dos dados foi realizada considerando-se um experimento bifatorial (hastes e genótipos). Primeiro, a análise foi realizada para estudar o efeito do número de hastes (uma haste e duas hastes) sobre as variáveis. Posteriormente, a análise foi aplicada para estudar o efeito da haste secundária sobre as variáveis apenas nas plantas conduzidas com duas hastes. Esta última análise permitiu estudar a competição intra-específica devido ao crescimento da segunda haste na planta. Os dados obtidos foram submetidos à análise de variância, e as médias dos genótipos foram comparadas pelo teste de Tukey a 5\% de probabilidade de erro. Ajustou-se também um modelo não-linear sigmoidal do tipo logístico $\mathrm{y}=\mathrm{a} /(1+\exp (-(\mathrm{x}-\mathrm{b}) / \mathrm{c}))$ aos dados de índice área foliar (IAF) (y) e NF (x) na haste principal e na secundária em que a, b e c são coeficientes estimados com o programa Table Curve 2D, utilizando-se médias de oito plantas por tratamento (SINCLAIR et al., 2004; PIVETTA et al., 2007).

\section{RESULTADOS E DISCUSSÃO}

A temperatura média do ar durante o período experimental foi de $25,7^{\circ} \mathrm{C}$, com valores absolutos da temperatura mínima e máxima de 8,1 e $39,8^{\circ} \mathrm{C}$, respectivamente. Nos primeiros 18 dias após transplante (DAT), a temperatura média diurna do ar ficou abaixo da Tot em praticamente todos os dias. Após os 18 DAT, a temperatura ficou sempre acima da Tot para a cultura.

A relação entre o NF nas hastes principal e secundária com a STa foi linear para os três genótipos, com coeficientes de determinação superiores a 0,98 . Esse resultado mostra que o fator abiótico que mais influenciou a emissão de folhas em berinjela foi a temperatura do ar, fato também constatado por STRECK et al. (2005b) e PIVETTA et al. (2007), para as culturas de cártamo e tomate, respectivamente.

O efeito dos fatores principais (número de hastes e genótipo) para as variáveis filocrono, número final de folhas (NFF), diâmetro de fruto (DF), comprimento de fruto (CF), peso médio de fruto (PMF) e produtividade de frutos ( $\mathrm{PF})$, comparando-se as plantas com uma haste com as plantas com duas hastes, foi significativo. Com exceção do diâmetro e comprimento de frutos, o quadrado médio foi maior para o fator número de hastes do que para o fator genótipo, quando foram analisados os efeitos para as mesmas variáveis, mostrando a maior influência do primeiro fator. A interação genótipo $\mathrm{x}$ haste foi significativa apenas para a variável produtividade de frutos.

A variedade Comprida Roxa apresentou maior filocrono do que os híbridos Ciça e Napoli, que não diferiram entre si (Tabela 1). Essa diferença no filocrono foi de $1,5^{\circ} \mathrm{C}$ dia entre os genótipos (Comprida 
Tabela 1 - Filocrono $\left({ }^{\circ} \mathrm{C}\right.$ dia folha $\left.{ }^{-1}\right)$, número final de folhas por planta (NFF), diâmetro do fruto (DF, cm), comprimento do fruto (CF, cm) e peso médio de frutos (PMF, g) de berinjela em três genótipos conduzidos com uma (H1) e duas hastes (H2), quando não houve interação significativa entre genótipo e haste, cultivados em estufa plástica. Santa Maria, RS, 2007.

\begin{tabular}{|c|c|c|c|c|c|}
\hline Genótipos & Filocrono & NFF & DF & $\mathrm{CF}$ & PMF \\
\hline ROXA & $18,2 \mathrm{a}$ & $49 c$ & $8,7 a$ & $13,1 b$ & $456,1 \mathrm{a}$ \\
\hline CIÇA & $17,1 b$ & $51 b$ & $7,0 \mathrm{~b}$ & $16,7 a$ & $386,6 b$ \\
\hline NAPOLI & $16,7 b$ & $53 a$ & $7,2 b$ & $17,5 a$ & 409,6ab \\
\hline \multicolumn{6}{|l|}{ № hastes } \\
\hline H1 & $22,3 a$ & $39,4 b$ & $7,8 a$ & $16,2 \mathrm{a}$ & 449,3а \\
\hline $\mathrm{H} 2$ & $12,4 \mathrm{~b}$ & 63,3a & $7,5 b$ & $15,4 \mathrm{~b}$ & $385,5 b$ \\
\hline Média geral & 17,4 & 51,4 & 7,7 & 15,8 & 417,3 \\
\hline $\mathrm{CV} \%$ & 3,1 & 2,1 & 4,3 & 5,3 & 12,1 \\
\hline
\end{tabular}

Médias não seguidas por mesma letra diferem entre si pelo teste Tukey a 5\% de probabilidade de erro.

Roxa e Napoli). Isso mostra que o genótipo Comprida Roxa necessita de maior soma térmica para emitir uma folha que os demais genótipos. O NFF foi maior para o híbrido Napoli e menor para a variedade Comprida Roxa. Os frutos desse genótipo apresentaram diâmetro maior e comprimento menor (Tabela 1). O peso médio de frutos foi maior na cultivar ‘Comprida Roxa’ e menor no híbrido Ciça.

Para a variável produtividade de frutos, observou-se que a berinjela produziu mais quando conduzida com duas hastes por planta. O genótipo mais produtivo é Ciça, conduzido com duas hastes (Tabela 2), com produtividade de frutos 32,8 e 21,4\% maior que os genótipos Comprida Roxa e Napoli, respectivamente. A menor produtividade foi da cultivar 'Comprida Roxa', conduzida com duas hastes por planta, o que pode ser devido ao fato desta ser de polinização aberta, enquanto as outras duas são híbridas. Esse resultado também mostra o maior

Tabela 2 - Produtividade de frutos (PF, $\mathrm{kg} \mathrm{ha}^{-1}$ ) de berinjela em três genótipos conduzidos com uma (H1) e duas hastes (H2), quando houve interação significativa entre genótipo e haste, cultivados em estufa plástica. Santa Maria, RS, 2007.

\begin{tabular}{lll}
\hline Genótipos & \multicolumn{1}{c}{ H1 } & H2 \\
& & \\
& & \\
ROXA & $37191,0 \mathrm{Aa}$ & $50258,7 \mathrm{Cb}$ \\
CIÇA & $37670,0 \mathrm{Aa}$ & $74823,7 \mathrm{Ab}$ \\
NAPOLI & $36817,5 \mathrm{Aa}$ & $59560,0 \mathrm{Bb}$ \\
& & \\
Média geral & 49385,2 & \\
CV \% & 8,8 & \\
\hline
\end{tabular}

Médias não seguidas por mesma letra maiúscula na vertical e minúscula na horizontal diferem entre si pelo teste Tukey a 5\% de probabilidade de erro. potencial produtivo do híbrido Ciça em relação ao híbrido Napoli em ambiente protegido, sendo este último o genótipo mais cultivado em área atualmente, especialmente em São Paulo (ANTONINI et al., 2002). Segundo RIBEIRO \& REISFSCHNEIDER (1999), o híbrido Ciça, lançado em 1991, apresenta boa aceitação pelos produtores rurais devido à maior precocidade, produtividade e qualidade de frutos e resistência a doenças. A cultivar 'Comprida Roxa' teve maior peso médio de frutos, não diferindo do hibrido Napoli. Isso também foi verificado por ANTONINI et. al. (2002), os quais relataram que os genótipos de polinização aberta apresentaram maior massa de frutos e menor número de frutos por planta em relação aos híbridos. As plantas conduzidas com uma haste apresentaram maior peso médio de frutos do que as conduzidas com duas hastes, possivelmente porque as plantas com uma haste apresentam menor competição intra-específica por fotoassimilados e nutrientes.

Comparando-se as variáveis medidas na haste principal com as medidas na haste secundária (das plantas com duas hastes), não houve interação significativa entre o número de hastes e os genótipos. Para o filocrono nas plantas conduzidas com duas hastes, houve diferença significativa apenas para genótipos (Comprida Roxa teve maior filocrono que os dois híbridos), sendo de $2,1^{\circ} \mathrm{C}$ dia folha ${ }^{-1}$ a diferença máxima entre o filocrono dos genótipos (Tabela 3).

O fato de o filocrono da HP não diferir do filocrono da HS (Tabela 3) está em desacordo com os resultados obtidos por PIVETTA et. al. (2007) para tomateiro, em que o filocrono na haste principal foi menor que em hastes laterais. Isso indica que, na berinjela, a competição entre as duas hastes não afeta a velocidade de emissão de folhas na HS.

Para as variáveis NFF, DF, CF, PMF e PF, a comparação entre genótipos não tem interesse prático, 
Tabela 3 - Filocrono ( ${ }^{\circ} \mathrm{C}$ dia), número final de folhas por planta (NFF), diâmetro do fruto (DF, cm), comprimento do fruto (CF, cm), peso médio de fruto (PMF, g) e produtividade de frutos $\left(\mathrm{kg} \mathrm{ha}^{-1}\right)$ de berinjela em três genótipos comparando a haste principal (HP) e a secundária (HS) das plantas conduzidas com duas hastes em estufa plástica. Santa Maria, RS, 2007.

\begin{tabular}{|c|c|c|c|c|c|c|}
\hline Genótipos & Filocrono & NFF & DF & $\mathrm{CF}$ & PMF & $\mathrm{PF}$ \\
\hline ROXA & $24,7 \mathrm{a}$ & $30,2 b$ & $8,5 a$ & $12,8 b$ & $411,1 \mathrm{a}$ & $25129,9 b$ \\
\hline CIÇA & $22,6 b$ & $31,9 a$ & $6,9 b$ & $16,6 a$ & 366,9a & $37413,0 a$ \\
\hline NAPOLI & $22,6 b$ & $32,4 a$ & $7,0 \mathrm{~b}$ & $17,0 \mathrm{a}$ & $356,7 a$ & 29775,0b \\
\hline \multicolumn{7}{|l|}{ Haste } \\
\hline HP & $23,1 \mathrm{a}$ & $38,9 a$ & $7,5 \mathrm{a}$ & $15,8 \mathrm{a}$ & $406,2 a$ & $38317,8 a$ \\
\hline HS & $23,5 a$ & $24,1 b$ & $7,4 a$ & $14,9 \mathrm{~b}$ & $350,2 b$ & $23227,1 b$ \\
\hline Média geral & 23,3 & 31,5 & 7,5 & 15,4 & 378,3 & 30772,5 \\
\hline $\mathrm{CV} \%$ & 4,2 & 2,9 & 5,2 & 5,9 & 14,4 & 12,7 \\
\hline
\end{tabular}

Médias não seguidas por mesma letra na vertical diferem entre si pelo teste Tukey a 5\% de probabilidade de erro.

pois representa a média das duas hastes na planta. Entre as hastes, houve diferença significativa nessas variáveis entre a haste principal e a secundária, com exceção do DF (Tabela 3). A HP teve maior NFF, CF, PMF e PF que a HS, resultado que pode ser atribuído à competição intra-específica por fotoassímilados na planta, que são translocados preferencialmente para a haste principal.

Também foi realizada a análise estatística pela comparação dos resultados das plantas conduzidas com uma haste com os resultados da haste principal das plantas conduzidas com duas hastes. Com essa análise, verificou-se que somente houve diferença significativa para a variável diâmetro de frutos, revelando que o diâmetro foi maior nas plantas conduzidas com uma haste. Isso demonstra que a competição da haste secundária não diminui o potencial da planta, principalmente em relação à produtividade de frutos.

Os coeficientes das equações de estimativa do índice de área foliar (IAF) com base no NF acumulada foram similares para os três genótipos avaliados conduzidos com uma haste (Figura 1A, 1B e 1C),

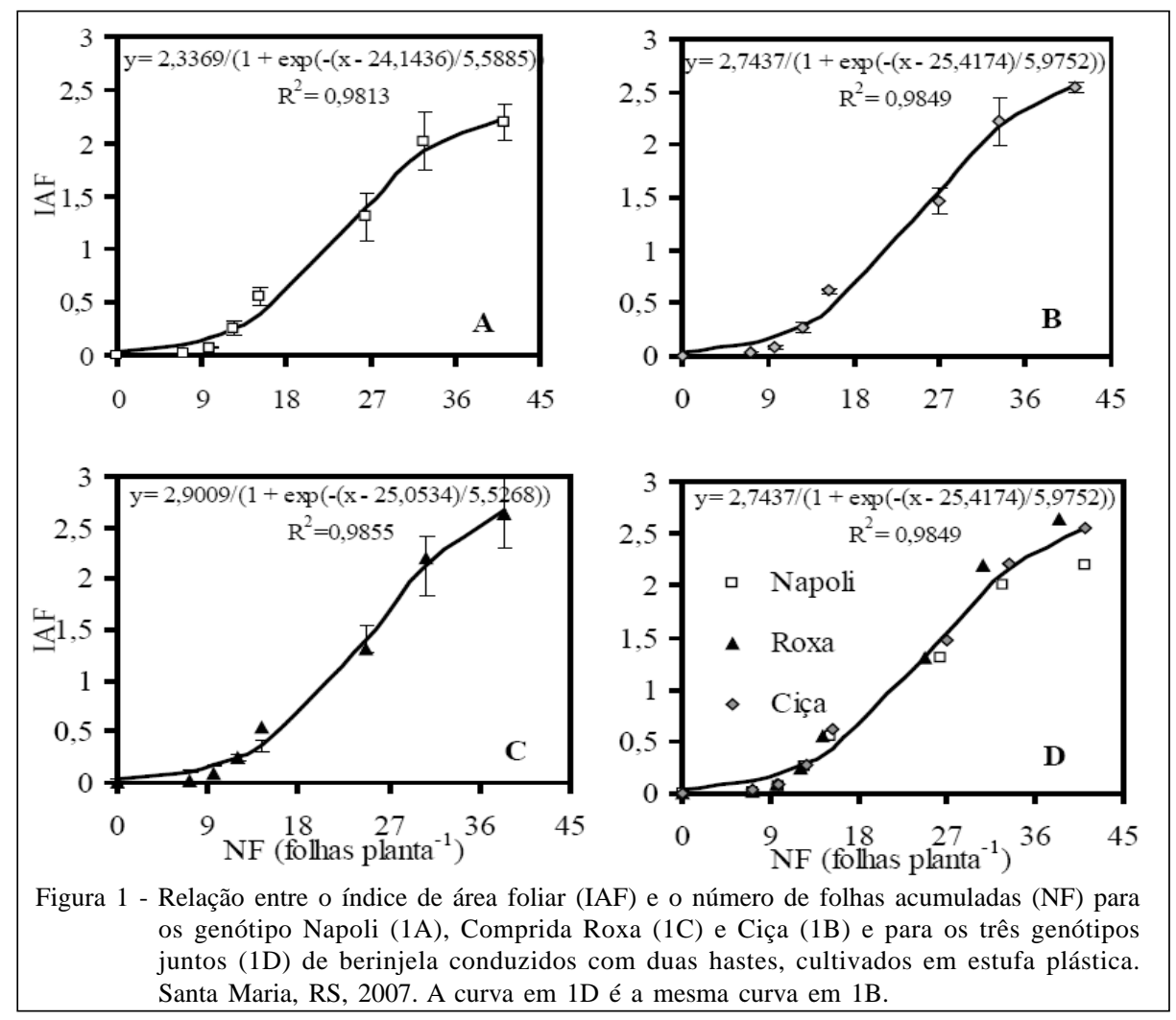

Ciência Rural, v.39, n.3, mai-jun, 2009. 
indicando uma relação similar entre IAF e NF nesses três genótipos. O modelo logístico com os coeficientes ajustados para o genótipo Ciça IAF= 2,7437/(1 + $\exp (-$ $(\mathrm{x}-25,4174) / 5,9752))$ descreve a tendência dos outros dois genótipos (Figura 1D). PIVETTA et al. (2007) também observam resposta similar de IAF com NF para dois genótipos de tomateiro. Tal resposta foi descrita por um único modelo matemático. Esses resultados indicam uma alternativa para estimar o índice de área foliar da berinjela sendo necessário apenas contar o número de folhas da planta, o que é uma vantagem em relação ao método tradicional, que precisa medir a largura das folhas (COSTA et al., 2007), demandando mão-de-obra e tempo.

\section{CONCLUSÕES}

A produtividade de frutos de berinjela é maior em plantas conduzidas com duas hastes. Além disso, a produtividade difere entre genótipos.

A presença da haste secundária na planta de berinjela resulta em competição intra-específica, afetando o filocrono, o número final de folhas e os componentes de produtividade de frutos nessa haste, comparada à haste principal.

A área foliar da berinjela pode ser estimada por meio do número de folhas acumuladas na planta.

\section{REFERENCIAS}

ANTONINI, A.C.C. et. al. Capacidade produtiva de cultivares de berinjela. Horticultura Brasileira, v.20, n.4, p.646- 648, 2002.

CAMARGOS, M.I. Produção e qualidade de tomate longa vida em estufa, em função do espaçamento e do número de cachos por planta. 1998. 68f. Dissertação (Mestrado em Fitotecnia) - Universidade Federal de Viçosa.

COSTA, M. da. et al. Modelos de determinação não destrutiva da área foliar da berinjela cultivada em estufa plástica. In: CONGRESSO BRASILEIRO DE AGROMETEOROLOGIA, 15., 2007, Aracaju-SE. Anais... Aracaju: Sociedade Brasileira de Agrometeorologia, 2007. CD-ROOM.

FILGUEIRA, F.A.R. Agrotecnologia moderna na produção e comercialização de hortaliças. Novo Manual de olericultura. Viçosa: UFV, 2000. 4 02p.

FONTES, P.C.R. et al. Produção e rentabilidade da cultura do tomateiro, afetadas pela fertilização e pelo sistema de condução. Revista Ceres, v.34, n.134, p.355-365, 1987.

GILMORE, E.C. Jr.; ROGERS, J.S. Heat units as a method of measuring maturity in corn. Agronomy Journal, v.50, n.10, p.611-615, 1958.

HERMES, C.C. et al. Emissão de folhas de alface em função da soma térmica. Revista Brasileira de Agrometeorologia, v.9, n.2, p.269-275, 2001.
HODGES, T.F. Predicting crop phenology. Boca Raton: CRC, 1991. 233p.

KÜRKLÜ, A. et al. Effects of temperature and time of harvest on the growth and yield of Aubergine (Solanum melongena L.). Agriculture and Forestry, v.22, p.341-348, 1998.

OLIVEIRA, V.R. et al. Efeito do número de hastes por planta e poda apical na produção classificada de frutos de tomateiro. Ciência e Prática, v.19, n.4, p.414-419, 1995.

PIVETTA, C.R. et al. Emissão e expansão foliar em três genótipos de tomateiro (Lycopersicon esculentum Mill.). Ciência Rural, v.37, n.5, p.1274-1280, 2007. Disponível em: http://www.scielo.br/ scielo.php? script=sci_arttext\&pid=S010384782007000500009\&lng=en\&nrm=iso\&tlng=pt. Doi: 10.1590/ S0103-84782007000500009.

POERSCHKE, P.R.C. et al. Efeito de sistemas de poda sobre o rendimento do tomateiro cultivado em estufa de polietileno. Ciência Rural, v.25, n.3, p.379-384, 1995.

RIBEIRO, C.S.C; REISFSCHNEIDER, F.J.B. Avaliação do híbrido de berinjela 'Ciça' por produtorese técnicos. Horticultura Brasileira, v.17, n.1, p.49-50, 1999.

RUSSELE, M.P. et al. Growth analysis based on degree days. Crop Science, v.24, n.1, p.28-32, 1984.

SILVA, D.J.H. et al. Produção de frutos de tomateiro (Lycopersicon esculentum Mill) em quatro sistemas de cultivo. Revista Ceres, v.44, n.252, p.129-141, 1997.

SINCLAIR, T.R. et al. Sugarcane leaf area development under field conditions in Florida, USA. Field Crops Research, v.88, n.1, p.171-178, 2004. Disponível em: http:// w w w. s c i e $\mathrm{n}$ c e d i r e c t. c o m / science?_ob=ArticleURL\&_udi=B6T6M-4BRSG03-

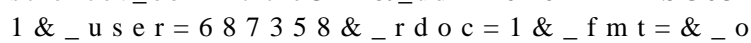
rig = search \&_sort $=$ d \& vie w = c \&_a c c t $=$ C 0000 $37899 \&$ \& v r s i o n $=1$ \&_ u r l V e r s i o $\mathrm{n}=0$ \&_userid=687358\&md5=602c3d58a15dd439b07e37b9c8cbfd82. Doi: $10.1016 /$ j.fcr.2003.12.005.

STRECK, N.A. et al. Incorporating a chronology response into the prediction of leaf appearance rate in winter wheat. Annals of Botany, v.92, p.181-190, 2003.

STRECK, N.A. et al. Estimativa do plastocrono em meloeiro (Cucumis melo L.) cultivado em estufa plástica em diferentes épocas do ano. Ciência Rural, v.35, n.6, p.1275-1280, 2005a. Disponível em: http:/www.scielo.br/scielo.php?script=sci_arttext\&pid=S010384782005000600008\&lng=en\&nrm=iso\&tlng=pt. Doi: 10.1590/ S0103-84782005000600008.

STRECK, N.A. et al. Estimating leaf appearance and phyllochron in safflower (Carthamus tinctorius L.). Ciência Rural, v.35, n.6, p.1448-1450, 2005b. Disponível em: http://www.scielo.br/ scielo.php? script=s ci_art text\&pid=s 0103 84782005000600036\&lng=en\&nrm=iso\&tlng=en. Doi: 10.1590/ S0103-84782005000600036. 
UZUN, S. The quantitative effects of temperature and light on the number of leaves preceding the first fruiting inflorescence on thestem of tomato (Lycopersicon esculentum Mill.) and aubergine (Solanum melongena L.). Scientia Horticulturae, n.109, p.142-146, 2006.

WILHELM, W.W.; McMASTER, G.S. Importance of the phyllochron in studyng development and growth in grasses Crop Science, v.35, n.1, p.1-3, 1995.

XUE, Q. et al. Predicting leaf appearance in field-grown winter wheat: evaluating linear and non-linear models. Ecological
Modelling, v.175, p.261-270, 2004. Disponível em: http:// w w w. s c i e n c e d i r e c t. c o m / science?_ob=ArticleURL\&_udi=B6VBS-4BJX1HYJ\&_user $=687358 \&$ \& rdoc $=1 \&$ \& $\mathrm{mt}=\&$ \&_orig $=$ search \& _ s o r t $=$ d \& vi e w $=$ c \& _ a c c t $=$ C 000037899 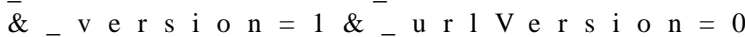 \&_userid=687358\&md5=5ee5e2b5d6f7ce4d3b0bd01537946560. Doi: 10.1016/j.ecolmodel.2003.10.018.

YI, J. A preliminary study on Inheritance of heat tolerance on eggplant. Beijin: Asian Regional Center, 1999. 6p. (Technical Report, 1999). 